\title{
3.5 Comparison of functional ability in juvenile idiopathic arthritis, juvenile dermatomyositis, juvenile systemic lupus erythematosus and healthy controls. An analysis of the PRINTO database
} G Filocamo*, S Meiorin*, C Saad-Magalhães, A Pistorio, A Ravelli, E Cortis, D Mihaylova, M Alessio, O Arguedas, S Garay, A Martini, N Ruperto and for the Paediatric Rheumatology International Trials Organisation (PRINTO)

Address: IRCCS Istituto G. Gaslini, Genoa, Italy

* Corresponding authors

from 15th Paediatric Rheumatology European Society (PreS) Congress London, UK. 14-17 September 2008

Published: 15 September 2008

Pediatric Rheumatology 2008, 6(Suppl I):S7 doi:I0.I 186/I546-0096-6-SI-S7

This abstract is available from: http://www.ped-rheum.com/content/6/SI/S7

(c) 2008 Filocamo et al; licensee BioMed Central Ltd.

\section{Objective}

To assess and compare functional ability, as measured by the Childhood Health Assessment Questionnaire (CHAQ), in juvenile idiopathic arthritis (JIA), juvenile dermatomyositis (JDM), juvenile systemic lupus erythematosus (JSLE) and healthy controls.

\section{Methods}

Patients with active JIA, JDM and JSLE, C-HAQ availability and less than 18 years were compared to healthy children. All patients were also evaluated for demographic and disease activity parameters at baseline and after 6 months.

\section{Results}

4,624 subjects (613 JIA, 277 JDM, 531 JSLE, and 3,203 healthy children) were included. Baseline functional disability was higher in patients with JDM (mean \pm SD C-HAQ $1.7 \pm 0.9)$, followed by JIA $(1.2 \pm 0.8$ JIA $)$, and JSLE $(0.8 \pm$ $0.9)$ and healthy control $(0.1 \pm 0.3)$. The C-HAQ score correlated moderately with the physical well being scale of the Child Health Questionnaire (CHQ PhS) for all 3 diseases and with the number of active joints in JIA, and with the Disease Activity Score (DAS) in JDM and poorly with JSLE disease activity variables. Common predictors of persistence of poor functional ability (C-HAQ $\geq 1.27, \geq 1, \geq$ 0.25 for JIA, JDM and JSLE respectively) were poor baseline C-HAQ and younger age at onset; other predictors were ANA negativity, and low CHQ-PhS for JIA, higher JSLE disease activity, and poor CHQ PhS and longer disease duration for JDM.

\section{Conclusion}

Baseline functional ability was poorer in active JDM than in JIA, and JSLE. A poor baseline C-HAQ and younger age at onset are predictors of persistence of poor functional ability despite treatment in either JIA, JDM and JSLE. 